\title{
Antiphospholipid syndrome presenting as progressive neuropsychiatric disorders: two case reports
}

This article was published in the following Dove Press journal:

Neuropsychiatric Disease and Treatment

22 May 2013

Number of times this article has been viewed

\author{
Chien-Hsun $\mathrm{Li}^{1-3}$ \\ Mei-Chuan Chou ${ }^{2,4}$ \\ Ching-Kuan $\mathrm{Liu}^{2,3}$ \\ Chiou-Lian Lai ${ }^{2,3}$ \\ 'Department of Neurology, Fooyin \\ University Hospital, Pingtung, \\ Taiwan; ' 2 Department of Neurology, \\ Kaohsiung Medical University \\ Hospital, Kaohsiung, Taiwan; \\ ${ }^{3}$ Department of and Master's Program \\ in Neurology, School of Medicine, \\ College of Medicine, Kaohsiung \\ Medical University, Kaohsiung, Taiwan; \\ ${ }^{4}$ Department of Neurology, Kaohsiung \\ Municipal Ta-Tung Hospital, Kaohsiung, \\ Taiwan
}

\begin{abstract}
The antiphospholipid syndrome (APS) is a rare form of autoimmune coagulopathy. In this syndrome, the most common neurologic abnormality is transient ischemic attack. This can be easily overlooked if a patient presents with progressive neuropsychiatric disorders, such as depression or dementia. We report two cases of young women, aged 35 and 22 years, presenting with progressive depression and mental decline over a certain period. The neuropsychological diagnoses of the two patients were, respectively, dementia with disinhibition and borderline dementia with depression. Brain magnetic resonance imaging showed multiple old infarcts with encephalomalacia in the former case, and only one cortical hemorrhagic infarction, over the right temporoparietal lobe, observed in the latter case. The outcomes of the two cases were also very different. Progressive neuropsychiatric disorders are increasingly observed in the young; therefore, APS and other autoimmune diseases should be considered during the differential diagnosis. Brain imaging examinations may prevent a delay in the detection of a structural lesion and facilitate the early intervention with good prognosis. Careful investigations by experts from different disciplines are always encouraged in complicated cases.
\end{abstract}

Keywords: autoimmune disease, brain imaging, dementia, depression, cerebrovascular disease

\section{Introduction}

The incidences of neuropsychiatric manifestations, like affective disorder or depression, are increasingly reported in general psychiatric and neurological practices, due to high-stress lifestyle. There are many contributing psychological, neuropsychiatric, and medical factors that should be investigated. The antiphospholipid syndrome (APS), also known as lupus anticoagulant syndrome or anticardiolipin antibody syndrome, is a rare form of autoimmune coagulopathy. ${ }^{1,2}$ APS is characterized and diagnosed by recurrent vascular thrombosis or pregnancy-related morbidity, in the presence of circulating antiphospholipid (APL) antibodies. It is usually observed in young adults with, the most common neurologic manifestation being transient ischemic attack; other manifestations include stroke, seizure, or acute encephalopathy. ${ }^{1,2}$ It can be easily overlooked if the patient presents with progressive neuropsychiatric disorders, such as depression or dementia. Herein, we report two young women with APS who presented with similar neuropsychiatric disorders but different radiological manifestations.

\section{Case reports Case I}

A 35-year-old female experienced progressive mental decline and depression for more than 2 years. Initially she was treated at a psychiatric outpatient department (OPD), for 1 year. 
The diagnosis was depression, and accordingly, antidepressants were administered. The depressive symptoms improved, but cognitive impairment did not show improvement; hence, she was referred to the neurological OPD. Initial laboratory analysis revealed elevated erythrocyte sedimentation rate (ESR) $(312 \mathrm{~mm} / \mathrm{h})$ and positive antinuclear antibody (ANA) test (1:80). Dementia was suspected, based on the clinical presentation, and she was admitted for further examination. Her history revealed four episodes of fetal abortion and diagnosis of APS 3 years previously, by medical record; she had discontinued treatment for APS for 2 years.

Neurological examination revealed the impairment of some cognition and mild unsteady gait. Laboratory findings indicated anemia (hemoglobin: $11.3 \mathrm{~g} / \mathrm{dL}$ ), thrombocytopenia (platelet count: $76000 / \mu \mathrm{L}$ ), and abnormal coagulation function (partial thromboplastin time [PTT]: 73.4/29.3 s; prothrombin time [PT]: 11.2/10.8 s; international normalized ratio [INR]: 1.16). Her autoimmune profiles were positive for anticardiolipin antibody (ACL), lupus anticoagulant antibody (LAC), ANA, rheumatoid arthritis factor (RA), APL immunoglobulin (Ig)G and IgM, anti-Ro, and showed decreased complement protein (C)3 and $\mathrm{C} 4$ levels (Table 1). Other related blood tests and cerebrospinal fluid (CSF) findings were normal results. The findings of brain magnetic resonance imaging (MRI) were multiple old infarcts with encephalomalacia in bilateral cerebral hemispheres and the left cerebellar hemisphere (Figure 1).

Table I Contributory autoimmune profiles of the two cases

\begin{tabular}{|c|c|c|c|}
\hline \multirow[t]{2}{*}{ Parameters (blood) } & \multicolumn{2}{|l|}{ Data } & \multirow{2}{*}{$\begin{array}{l}\text { Normal } \\
\text { range** }^{* *}\end{array}$} \\
\hline & Case I & Case 2 & \\
\hline ESR & 312 & 26 & $<30(\mathrm{~mm} / \mathrm{h})$ \\
\hline $\mathrm{ACL}$ & $+(19.49)$ & $+(87.2 \mathrm{I})$ & $<15(\mathrm{GPL} / \mathrm{mL})$ \\
\hline LAC & $+(1.46)$ & $+(1.63)$ & $<\mathrm{I} .2$ ratio \\
\hline APL IgG & $+(36.6)$ & NA & $<15$ (GPL) \\
\hline APL IgM & $+(17.8)$ & $-(7.7)$ & $<15$ (MPL) \\
\hline$A \beta 2 G P-I$ & $+($ weak $)$ & $+(76.6)$ & $<20$ (SGU) \\
\hline ANA & $+(1: 80)$ & $-(<\mathrm{I}: 40)$ & $<\mathrm{I}: 40$ \\
\hline AdsDNA & $-(1.3)$ & NA & $<25(\mathrm{IU} / \mathrm{mL})$ \\
\hline RA & $+(46.7)$ & $-(<20)$ & $<20(\mathrm{IU} / \mathrm{mL})$ \\
\hline Anti-Ro & $+(227)$ & $-(<0.3)$ & $<7$ (EliAU/mL) \\
\hline Anti-La & $-(6)$ & $-(<0.3)$ & $<7$ (EliAU/mL) \\
\hline $\mathrm{C} 3$ & 74 & 133 & $83 \sim 125(\mathrm{mg} / \mathrm{dL})$ \\
\hline $\mathrm{C4}$ & 13 & 23.9 & I7 33 (mg/dL) \\
\hline
\end{tabular}

Note: ** These data are indicated from the clinical laboratory of Kaohsiung Medical University Hospital. For case I and $2+$ indicates a positive result and - indicates a negative, normal result.

Abbreviations: A $32 \mathrm{GP}-\mathrm{I}$, anti- $\beta 2$ glycoprotein-I antibody; $A C L$, anticardiolipin antibody; AdsDNA, anti-double strain DNA antibody; ANA, antinuclear antibody; Anti-La, anti La antibody; Anti-Ro, anti Ro antibody; APL, antiphospholipid antibody; C, complement protein; ESR, erythrocyte sedimentation rate; Ig, immunoglobulin; LAC, lupus anticoagulant antibody; NA, not available; RA, rheumatoid arthritis factor.

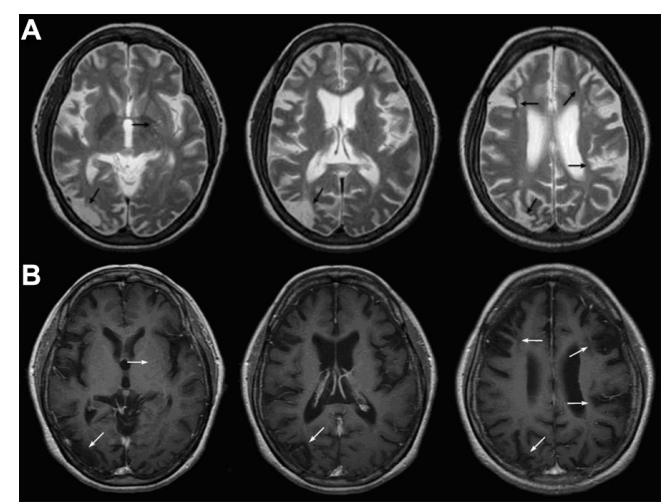

Figure I Case I: brain magnetic resonance imaging (MRI) showed old infarction and encephalomalacia lesions in the left lentiform nucleus, right temporal, occipital, and bilateral frontal and parietal lobes, with hyperintensity on T2-weighted imaging (A) and hypointensity without enhancement on postenhanced TI-weighted imaging (B).

Her cognitive ability screening instrument (CASI) score was 19 (cutoff value is 85 , below is abnormal result). ${ }^{3}$ The CASI-estimated mini-mental state examination (MMSECE) score was 9 (cutoff value is 25, below is abnormal result). The global clinical dementia rating (CDR) score was 1.0. Assessment with the neuropsychiatric inventory (NPI) indicated disinhibited behavior. According to the report of neuropsychological testing, the patient was diagnosed with dementia and disinhibition (Table 2). An electroencephalogram (EEG) examination showed normal findings.

The diagnosis of APS was confirmed after consultation with a rheumatologist. Warfarin was prescribed to control coagulopathy, along with a disease-modifying antirheumatic drug (hydroxychloroquine). However, we lost this patient to follow-up for unknown reason.

\section{Case 2}

A 22-year-old unmarried female developed progressively depressive mood over a period of 1 month. Gradually, her verbal output decreased, with incoherent speech, and she developed mild left upper limb weakness. She was brought to a psychiatric clinic, and the initial diagnosis was depression. Intermittent involuntary movements of her four limbs were observed for several days before her referral to the neurological OPD. Neurological examination revealed abulia and slight decrease in the muscle power of her left upper limb. Immediately brain computed topography (CT) was performed; the results revealed a hyperdense, gyriform lesion along the cortices of the right temporoparietal lobe, with perifocal edema (Figure 2A). Following this, she was transferred to a medical center hospital.

At the emergency department, the laboratory blood test revealed anemia (hemoglobin: $11.3 \mathrm{~g} / \mathrm{dL}$ ), thrombocytopenia 
Table 2 Results of the neuropsychological test

\begin{tabular}{llllll}
\hline & Attention & Language & Memory & Orientation & Visual \\
\hline $\begin{array}{lllll}\text { Neurobehavioral findings } \\
\text { Case I }\end{array}$ & $\mathrm{ABNL}$ & $\mathrm{ABNL}$ & $\mathrm{ABNL}$ & $\mathrm{ABNL}$ & $\mathrm{ABNL}$ \\
Case 2 & $\mathrm{ABNL}$ & $\mathrm{NL}$ & $\mathrm{ABNL}$ & $\mathrm{ABNL}$ & $\mathrm{NL}$ \\
& Abstract thinking & Judgment & Verbal fluency & Personality/behavior & Depression \\
\hline Case I & $\mathrm{ABNL}$ & $\mathrm{ABNL}$ & $\mathrm{ABNL}$ & Disinhibition & $\mathrm{NL}$ \\
Case 2 & $\mathrm{NL}$ & $\mathrm{NL}$ & $\mathrm{NL}$ & Delusion & Borderline \\
\hline
\end{tabular}

Abbreviations: $A B N L$, abnormal; NL, normal.

(platelet count: $75000 / \mu \mathrm{L}$ ), and normal coagulation function (PTT: 30.6/28.6 s; PT: 10.9/10.7 s; INR: 1.04). The brain CT findings were discussed with a neurosurgeon, and arteriovenous malformation (AVM) with hemorrhage was suspected (Figure 2). The brain MRI finding was subacute to chronic infarction with hemorrhagic transformation in the right temporoparietal lobe; the differential diagnosis was encephalitis with petechial hemorrhage (Figure 3). Then, the patient was transferred to the neurological department to rule out encephalitis.

Further laboratory findings indicated an abnormal autoimmune function (positive ACL, LAC, and anti- $\beta 2$ glycoprotein-I antibody, and elevated C3 level) (Table 1), and abnormal thyroid function. Other related blood tests and CSF findings were within normal limits. The CASI and MMSE-CE scores were 85 and 25, respectively. The global CDR score was 0.5 . The NPI assessment revealed depression and delusion. Based on the neuropsychological testing, borderline dementia with depression and delusion were diagnosed (Table 2). An EEG examination showed normal background activity with an intermittent theta wave over the right hemisphere.

The final diagnosis was established after consulting the rheumatologist; the APS was complicated by cerebral hemorrhagic infarction accompanied by borderline

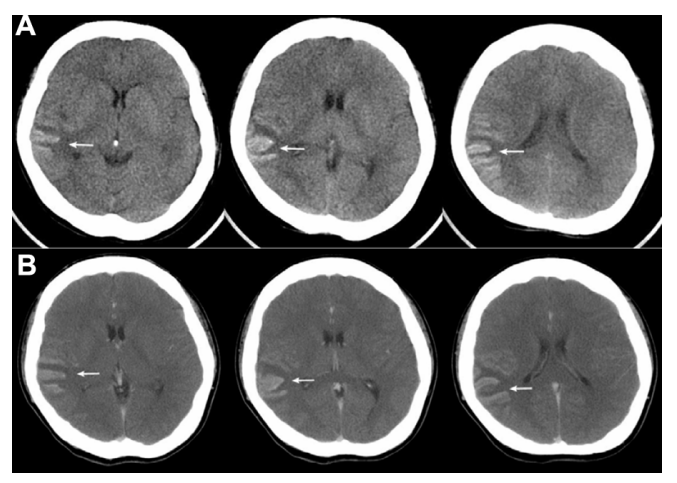

Figure 2 Case 2: brain computed topography (CT) without contrast (A) and postcontrast (B) showed hyperdense lesions with mild gyriform enhancement along the cortices of the right temporoparietal lobe. dementia, depression, suspected seizure, and concomitant hyperthyroidism. The follow-up brain CT 17 days later revealed encephalomalacia in the right temporoparietal lobe, with partial resolution of hemorrhagic transformation. Because of the bleeding tendency underlying the recent hemorrhagic infarction, we prescribed acetylsalicylic acid instead of warfarin for temporary stroke prevention. These neuropsychiatric disorders gradually recovered after 1 month.

\section{Discussion}

In the two young patients with initial presentations of progressive depression and mild cognitive impairment, it is likely that an organic brain lesion could have been overlooked during diagnosis. Notably, detailed review and investigation should be performed when patients present with obscure stress events, failed medication response, and persistent neuropsychiatric symptoms. The common differential diagnoses of young patients with neuropsychiatric disorders or dementia include structural disease, autoimmune encephalopathy, vasculopathies, psychiatric

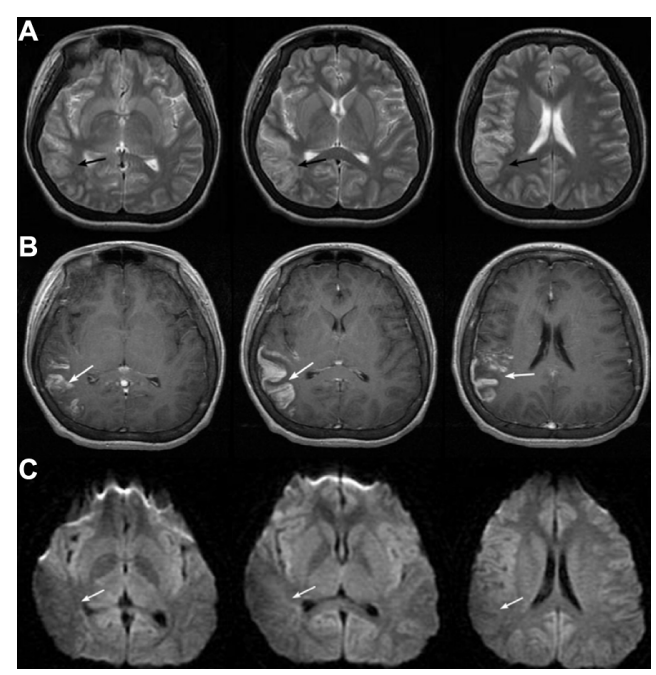

Figure 3 Case 2: brain magnetic resonance imaging (MRI) showed subacute to chronic infarction with hemorrhagic transformation lesions along the gyri of the right temporoparietal lobe, with mild hyperintensity and hypointensity linear lesions on T2-weighted imaging (A), gyriform enhancement on postenhanced TI-weighted imaging (B), and mild hypointensity on diffusion-weighted imaging (C). 
illness, etc. The reversible and treatable underlying disorders should be given priority. ${ }^{4}$

Central nervous system (CNS) involvement is a prominent feature of APS; many acute neurological manifestations have been described in APS patients. However, there are limited reports on the psychiatric manifestations associated with APS, such as depression and mania. ${ }^{5}$ Primary APS is considered a rare autoimmune disease and secondary APS is closely related to systemic lupus erythematosus (SLE), hematologic disorders, infection, and some medications. In the case of SLE, there is an association between neuropsychiatric symptoms and specific antibodies; ${ }^{6,7}$ in particular, APL antibodies have been associated with stroke, vascular dementia, and epilepsy. ${ }^{8-10}$

In our first case, the patient did not completely satisfy the diagnostic criteria of probable SLE, after consultation with the rheumatologist. ${ }^{11}$ However, SLE might be diagnosed at a later stage in the clinical course, and hence, regular follow-up visits were strongly advised. In the second case, the brain images were difficult to interpret in the first reading. Unlike the usual images of acute stroke, those of APS might mimic tumor mass, vascular malformation, and inflammatory lesion at the subacute to chronic stage. It is necessary to conduct careful investigations, with the cooperation of experts from various disciplines.

Another important inference from these two case studies is that APS might present as similar neuropsychiatric disorders (ie, depression or dementia) arising from different cerebrovascular lesions involving the contributory circuits and resulting in vascular depression and vascular dementia. The resulting outcomes may be very different. Early intervention will allow good prognosis.

Regarding the treatment of APS-related neuropsychiatric disorders, the symptomatic control of vascular complications and management of underlying autoimmune coagulopathy (disease-modifying antirheumatic drugs and anticoagulation agents) are suggested. For vascular depression, the antidepressant of selective serotonin reuptake inhibitor (SSRI) type might be the considered choice because of its lesser influence on the cardiovascular system. On the other hand, the circulation-promoting agents and acetylcholinesterase inhibitors could be useful for vascular dementia, according to the patient's clinical status. Additionally,

Neuropsychiatric Disease and Treatment

\section{Publish your work in this journal}

Neuropsychiatric Disease and Treatment is an international, peerreviewed journal of clinical therapeutics and pharmacology focusing on concise rapid reporting of clinical or pre-clinical studies on a range of neuropsychiatric and neurological disorders. This journal is indexed on PubMed Central, the 'PsycINFO' database and CAS. many nonpharmacological interventions are available and are suggested as concomitant therapy for intensive care of neuropsychiatric disorders.

\section{Conclusion}

Because the incidences of progressive neuropsychiatric disorders in the young are increasing, we have underscored the clinical relevance of the differential diagnoses of systemic autoimmune diseases with CNS involvement, such as APS. Brain imaging is an essential tool to prevent any delay in the detection of structural lesions and facilitate the early intervention with good prognosis. Careful investigations and the cooperation of multidisciplinary experts are always encouraged in complicated cases.

\section{Disclosure}

The authors report no conflicts of interest in this work.

\section{References}

1. Ropper AH, Samuels MA. Adams and Victor's Principles of Neurology, 9th ed. New York, NY: McGraw-Hill; 2009.

2. Levine JS, Branch DW, Rauch J. The antiphospholipid syndrome. N Engl J Med. 2002;346(10):752-763.

3. Lin KN, Wang PN, Liu CY, Chen WT, Lee YC, Liu HC. Cutoff scores of the cognitive abilities screening instrument, Chinese version in screening of dementia. Dement Geriatr Cogn Disord. 2002;14(4):176-182.

4. Gómez-Puerta JA, Cervera R, Calvo LM, et al. Dementia associated with the antiphospholipid syndrome: clinical and radiological characteristics of 30 patients. Rheumatology (Oxford). 2005;44(1):95-99.

5. Raza H, Epstein SA, Pao M, Rosenstein DL. Mania: psychiatric manifestations of the antiphospholipid syndrome. Psychosomatics. 2008;49(5): $438-441$.

6. Greenwood DL, Gitlits VM, Alderuccio F, Sentry JW, Toh BH. Autoantibodies in neuropsychiatric lupus. Autoimmunity. 2002;35(2): 79-86.

7. Zandman-Goddard G, Chapman J, Shoenfeld Y. Autoantibodies involved in neuropsychiatric SLE and antiphospholipid syndrome. Semin Arthritis Rheum. 2007;36(5):297-315.

8. Asherson RA, Mercey D, Philips G, et al. Recurrent stroke and multiinfarct dementia in systemic lupus erythematosus: association with antiphospholipid antibodies. Ann Rheum Dis. 1987;46(8):605-611.

9. Fukui T, Kawamura M, Hasegawa Y, Kato T, Kaga E. Multiple cognitive impairments associated with systemic lupus erythematosus and antiphospholipid antibody syndrome: a form of progressive vascular dementia? Eur Neurol. 2000;43(2):115-116.

10. Cimaz R, Meroni PL, Shoenfeld Y. Epilepsy as part of systemic lupus erythematosus and systemic antiphospholipid syndrome (Hughes syndrome). Lupus. 2006;15(4):191-197.

11. Petri M. Review of classification criteria for systemic lupus erythematosus. Rheum Dis Clin North Am. 2005;31(2):245-254.

\section{Dovepress}

The manuscript management system is completely online and includes a very quick and fair peer-review system, which is all easy to use. Visit http://www.dovepress.com/testimonials.php to read real quotes from published authors. 